\title{
Linx
}

Revue des linguistes de l'université Paris X Nanterre

$12 \mid 2002$

"Comme la lettre dit la vie »

\section{SIDI BOU SAÏD, « village maraboutique », le «Saint-Paul de Vence » tunisien}

Geneviève Petiot

\section{(2) OpenEdition}

Journals

Édition électronique

URL : http://journals.openedition.org/linx/1308

DOI : 10.4000/linx.1308

ISSN : 2118-9692

Éditeur

Presses universitaires de Paris Nanterre

\section{Édition imprimée}

Date de publication : 1 octobre 2002

Pagination : $217-230$

ISSN : 0246-8743

\section{Référence électronique}

Geneviève Petiot, «SIDI BOU SAÏD, « village maraboutique », le « Saint-Paul de Vence » tunisien », Linx [En ligne], 12 | 2002, mis en ligne le 10 octobre 2012, consulté le 19 avril 2019. URL : http:// journals.openedition.org/linx/1308; DOI : 10.4000/linx.1308

Ce document a été généré automatiquement le 19 avril 2019

Département de Sciences du langage, Université Paris Ouest 


\title{
SIDI BOU SAÏD, « village maraboutique », le « Saint-Paul de Vence » tunisien
}

\author{
Geneviève Petiot
}

1 Je me propose ici, à partir d'une analyse de discours de guides touristiques français consacrés à la Tunisie, en l'occurrence au village de Sidi-Bou-Saïd, proche de Tunis, d'en mettre en lumière deux fonctionnements caractéristiques: le syncrétisme opéré entre données tunisiennes et islamiques d'une part, françaises et chrétiennes de l'autre, ainsi que la construction « française » de ce syncrétisme.

2 Les visites proposées par ces ouvrages sont en effet guidées et orientées par un regard français, soucieux de lire les traces laissées dans ce lieu; même les exotismes, paradoxalement, participent de ce regard, ainsi que les évocations historiques des héritages complexes de l'actuelle Tunisie.

3 Le corpus ${ }^{1}$ date, pour l'essentiel, de la fin du $\mathrm{XX}^{\mathrm{e}}$ siècle. Mais le premier guide retenu est antérieur à l'indépendance du pays (1955) et aura valeur de contraste, par rapport au corpus de base. En dépit de leurs différences dues à leurs spécificités éditoriales ${ }^{2}$, les textes retenus présentent, peu ou prou, les caractéristiques dégagées ci-dessus, fondatrices d'un sens commun. Si le genre du guide touristique permet de réunir des textes distincts en fonction de leur objet (et référent) commun, c'est la recherche d'un fonctionnement énonciatif particulier, liée à l'analyse de discours, qui les constitue en corpus discursif.

4 A la lumière des études émerge en effet un discours de doxa: stéréotypes et comportement impliqués par le statut de touriste français à l'étranger, mais dans un pays dont les relations tissées avec la France au cours de l'histoire, ancienne (Croisades) et récente (les $\mathrm{XIX}^{\mathrm{e}}$ et $\mathrm{XX}^{\mathrm{e}}$ siècles), sont posées comme l'une des clés pour apprécier le village de Sidi-Bou-Saïd. La culture est ainsi convoquée, culture avant tout française, mais aussi européenne. Le touriste suit des itinéraires tracés par d'illustres prédécesseurs, écrivains et peintres. De surcroît se développe, de guide en guide, le récit, ignoré en 1955, d'une légende qui ferait de Saint Louis le saint musulman qui a donné son nom honoré en 
ce lieu. Le décor lui-même serait dû à l'intervention d'un riche mécène européen. C'est donc bien l'intervention répétée d'artistes non tunisiens qui donne vie et sens à ce qui est certes tunisien, mais dont l'essence serait modelée et forgée par des interventions extérieures. Lire et interpréter Sidi-Bou-Saïd, ce serait, à travers les arabismes manifestés notamment par des emprunts lexicaux et la notation d'objets typiques (à acheter ?), voir les traces de cet héritage européen et leur symbiose dans un décor digne des hauts lieux de la Côte d'Azur française.

5 Je m'attacherai donc à distinguer ce qui est le fait de tout guide : notations précises, indications et informations de tous ordres, ce qui constitue un discours de doxa : ce qu'il faut savoir, du moins ce dont on doit avoir entendu parler, et ce qui oriente la lecture du touriste: le point de vue manifesté notamment par les modalités d'énonciation et d'énoncé, les axiologiques et la représentation de discours autres, mais lesquels?

6 Ces études devraient me permettre de dégager la façon dont chaque guide répercute et amplifie certains traits déjà saillants dans les guides précédents, exploitant et maintenant la « mémoire discursive $»^{3}$, ainsi que l'image du touriste façonnée par ce discours.

\section{D'une pratique touristique à une doxa}

7 Les indications d'itinéraires, du temps nécessaire pour la visite et des lieux et monuments à voir font partie des données fournies par tout guide. Elles figurent bien évidemment dans le corpus retenu. Mais on relève qu'elles sont toutes situées à partir de Tunis, vers la côte nord, ce qui implique un ordre de visite posé comme allant de soi ; c'est Tunis qui est pris comme point de repère pour tout voyage en Tunisie, exception faite pour l'île de Djerba, qui possède son propre aérodrome international.

8 Les raisons pour lesquelles il faut se rendre à Sidi-bou-Saïd sont à la fois implicites : le sous-chapitre consacré au village signifie son importance, et explicitées, notamment par l'usage de nombreux axiologiques.

\subsection{Les axiologiques}

Accompagnant les descriptions objectives (les ruelles étroites et pentues, ...), ils qualifient aussi bien le site et le village que les monuments et constructions à repérer et visiter.

Site et village font en effet l'objet d'appréciations toutes porteuses de degré positif et du sème /beauté/, volontiers en relation avec/charme/ou encore /plaisir/: un site magnifique, un site admirable, panorama magnifique (GB1), avec l'écho de GB2 un tableau admirable, et Quel sortilège en ce lieu, qui redonne à charme, charmant, quelque chose du sens originel, le charme tranquille, l'incomparable bleu..., séduit avant nous, très belle vue, d'où la vue est splendide, une vue magnifique (GB2), à la perfection le village de charme. Ce joyau..., à la vue imprenable [...](GV), [Le village est célèbre] pour son incomparable beauté, l'un des plus beaux panoramas, une vue enchanteresse(B), Le plus pur, le plus beau village tunisien est certainement Sidi-Bou-Saïd, du village mythique (GG), son site remarquable[...] ce charmant village (GN), une excursion agréable, l'hôtel Sidi-Bou-Said magnifiquement situé (GGT), [y passer quelques heures] mais qui valent vraiment le coup, un véritable balcon sur la mer, "Perle blanche suspendue... », le plus fameux des villages tunisiens [où se mêlent] avec un rare bonheur, panorama magnifique (GR). 
11 Les constructions et les objets caractéristiques du village font eux aussi l'objet d'appréciations comparables : de jolies maisons mauresques (GB1), un élégant minaret (GB1, GV, GB2), les belles volières bleues et blanches ..., un jardin odorant... (GV), ses façades immaculées (adjectif à la fois descriptif et axiologique), un charme particulier (GB2), des maisons cubiques immaculées, les fameuses cages à oiseaux, les élégantes courbes ... des fenêtres, l'un des beaux hôtels (B), les fières maisons, aux murs d'une blancheur éclatante, jusqu'aux imposantes marches (GN), avec ses maisons étonnantes de blancheur, on se laisse éblouir par la blancheur des murs (avec un jeu sémantique par les traits [+concret] et [+abstrait] conjoints), le blanc et le bleu se complètent dans une harmonie[...], sous le charme de cette architecture, le Café..., un endroit exceptionnel(GR).

Les signes codés, spécifiques de certains guides, comme les étoiles, à la fois informent sur l'intérêt de la visite, mais aussi, par leur gradation laissée au choix du guide, participent de ces évaluations.

On le constate à ce relevé rendu fastidieux par la répétition - écho de guide en guide, les éléments informatifs sont pleinement ré-interprétés par des points de vue louangeurs, qui mettent le touriste consciencieux dans l'impossibilité de ne pas se rendre à Sidi-BouSaïd, et de ne pas reconnaître la justesse de ces appréciations ? Visiter et aimer le village est une obligation touristique. Modalité appréciative et emploi d'axiologiques participent ainsi à la mémoire discursive du genre " guide touristique ».

\subsection{Du bon usage d'exotismes}

14 Chaque guide distille des emprunts à l'arabe, permettant apparemment aux visiteurs de s'approprier des termes spécifiques de l'architecture et de la vie quotidienne.

15 La liste de ces emprunts n'est pas longue, et dans certains cas, renvoie à des exposés " savants ", situés dans les premières pages des ouvrages, et soucieux de contribuer, d'un point de vue didactique et culturel, à l'acquisition de connaissances par les touristes.

16 Les noms propres sont présents dans tous les guides; celui du village, et de son saint patron, bien sûr, parfois celui de la colline, le djebel Manâr, mais aussi ceux des hôtels, Dar Zarrouk, éventuellement Dar Saïd et Sidi Boufarès, ainsi que des maisons et cafés. Si les deux premiers se trouvent dans le cours de l'exposé consacré à la visite, les autres, qui ont valeur informative, sont répartis dans des rubriques différentes en fonction de l'organisation de chaque guide, propre à la conception d'une collection. Ainsi, l'existence et la disposition de telles rubriques diffèrent au sein d'une même maison d'édition ${ }^{4}$.

Les noms techniques jouent un rôle autre. Ils participent au tourisme proprement dit, en ce qu'ils permettent de prendre conscience des différences lexicales, mais surtout référentielles. Absent de GB1, qui parle à propos des palais de la Médina de Tunis, de "céramiques peintes» [zellige], les termes moucharabieh et zellige, éventuellement francisés par la marque finale de pluriel (GV, GB2), se trouvent dans les autres guides, à l'exception notable de B qui se contente de parler des « élégantes courbes en fer forgé des fenêtres des maisons ». Le ribat, précédé de sa traduction dans GV, est souvent signalé, tout comme la zaouia et le marabout, qui va caractériser Sidi-bou-Saïd. Comme village maraboutique (GV), ou encore la koubba (GB1); dans ce dernier guide, ces termes, tout comme le soufisme, volontiers évoqué par des successeurs, font l'objet de longs exposés dans les différents " Aperçus » qui ouvrent le guide et sont signés par des spécialistes des domaines. La description des cafés indiqués comme visite obligée, le Café des Nattes et/ou 
le café Chaabane, voire Chergui, inclut l'emprunt de la chicha, qui contribue à la couleur locale. Le malouf, parfois orthographié maluf, est l'un des éléments caractéristiques du village ${ }^{5}$, lié à un passé andalou encore présent en ces lieux. GR, enfin, ose un emploi, décalé par rapport aux relevés précédents : D'ailleurs, jusqu 'en 1820, l'accès au village, haut lieu de spiritualité, fut interdite aux roumis.

Ces mots étrangers, volontiers traduits ou glosés dans le discours, n'ont pas seulement une connotation d'exotisme; ils relèvent aussi de la connotation autonymique ${ }^{6}$, voire de la modalisation autonymique ${ }^{7}$.

La couleur locale est explicitée par les couleurs caractéristiques des maisons, l'incomparable "bleu Sidi Bou Saïd» (GB2), et la description des « hauts lieux» du village ; elle est évoquée par la présence des parfums (jasmin et chèvrefeuille notamment) exhalés par les jardins, non visibles, par le rappel des ruelles étroites et pentues, et l'encouragement à une promenade hors trajet courant, là où jouent et rôdent les chats (demi-sauvages) et les enfants, comprenant le cimetière, voire le phare. Le GG est le seul, dans ses notes, à évoquer l'usage au XIX ${ }^{\mathrm{e}}$ siècle des maâouma, vivifiant la représentation traditionnelle des femmes cachées à la vue.

20 Le soufisme, dont le saint patron du village se réclamait, est l'objet d'exposés plus ou moins longs et documentés ; GG est, de ce point de vue, le plus informatif. Puisant dans les textes de J. Duvignaud, il informe sur les processions, la recherche de la transe et de l'extase, tout en s'effaçant, en le représentant, devant un discours autre, discours pleinement autorisé. Ce faisant, c'est à un chercheur français et non aux sources du soufisme (écrites en arabe et traduites) qu'il puise citations et point de vue faisant autorité.

21 Ces emprunts, réemployés en situation, et ces informations, plus ou moins savantes, suggèrent des niveaux de visites, de lectures et d'approfondissements, laissés à la liberté des voyageurs, qui se font alors découvreurs, grâce à la présence insistante dans tous les guides, à l'exception de GB1, du charme et du « cachet » particulier de Sidi-bou-Saïd. Le « vu » est indice de « caché » à découvrir et apprécier.

\subsection{Une doxa}

Ces différentes caractéristiques formelles, communes, grosso modo (GB1 à part), aux différents guides retenus, ne se contentent pas de fournir des informations et des appréciations, ainsi que des connaissances partielles et liées au pays visité. Elles posent des descriptions, des désignations et des opinions indiscutables, que doivent s'approprier les lecteurs touristes. De même que des marques peuvent appeler leurs produits des «must », Sidi-Bou-Saïd est ainsi posé comme un «must » de tout voyage en Tunisie. De surcroît, contrairement aux visites de sites antiques comme Carthage, ou de musées comme celui du Bardo, la visite du village contraste agréablement avec ces taches fastidieuses (GGT).

Il s'agit aussi d'imposer une sorte d'image d'Epinal, un village de carte postale. Dans cette représentation, sont convoquées, outre les éléments relevés, les couleurs, ainsi que les impressions à ressentir au cours de la promenade.

Les axiologiques relevés ne manifestent pas seulement le point de vue du guide : ils sont à partager avec les visiteurs, puisqu'impressions et sentiments sont pré-dictés (« prédigérés ») : comment pourrait-on ne pas se rendre au charme imposé par les textes, les 
témoignages, les rappels historiques et culturels ? Là encore, GB1, par son strict point de vue informatif, s'oppose à tous ses successeurs.

Se développe ainsi une isotopie du secret et du mystère, due aux murs cachant la vie intime, qui a à voir avec la représentation stéréotypée des palais arabes dont les splendeurs sont cachées à la vue, des femmes voilées, des harems, ..., chers à l'évocation occidentale de l'Orient. Ainsi, dit GN, il faut s'éloigner du centre pour découvrir des ruelles un peu moins retapées et aseptisées à l'intention des touristes et des étrangers à demeure. Non seulement le guide crée "le bon » touriste, mais il lui dit comment ne plus être touriste pour trouver la vérité des lieux, qui, selon GG, semble(nt) ignorer les touristes, les habitants [sachant] se garder des regards indiscrets....

Les stéréotypes dégagés en construisent un autre, celui du touriste acharné à découvrir le « vrai » en s'aidant de ses lectures, qui déchiffrent pour lui le secret, et en s'autorisant un regard furtif (GB2). Bien que guidé, le visiteur se doit aussi de chercher à trouver par luimême le vrai visage qu'il lit. Les indications d'itinéraires, liées au genre même du guide, en prennent une autre valeur injonctive: c'est le chemin qu'il faut suivre pour atteindre le cœur du village.

\section{La convocation et la révision de l'histoire}

Tous les guides du corpus comprennent une sous-partie «histoire», soit intégrée, soit distincte des pages consacrées à la visite. Les grandes dates, vues notamment d'un regard français, y sont diversement appréhendées. Si le passé punique et le siège tenté par Louis IX sont rappelés par tous, certains (GGT notamment) vont jusqu'à évoquer la Reconquista et l'arrivée des Andalous, puis les passages des Espagnols et le rôle joué par les Turcs. Le passé de Sidi-Bou-Saïd est manifestement évolutif, du GB1 de 1955 au GR de 2002, puisque la présence de Saint-Louis apparaît sous des aspects inattendus. Mais Carthage est aussi évoquée, avec de curieuses variantes.

\subsection{Carthage et Sidi-Bou-Saïd}

Le djebel Manâr, sur les pentes duquel est construit Sidi-Bou-Saïd, domine, selon GB1 et GB2, le cap Carthage, nom qui ne figure ni sur les cartes de Tunisie ni dans les autres guides. En revanche, le nom et parfois l'histoire de la ville punique, en relation avec le village, sont évoqués par tous, à l'exception de GB1, la visite du site et des fouilles de Carthage faisant l'objet d'exposés particuliers.

Ainsi, pour GV, c'est l'occasion de parler des feux-signaux qui guidaient les galères puniques vers le port, rejoint sur ce point par GG qui s'interroge : le lieu semble chargé de densité religieuse : celle-ci remonte-t-elle aux Carthaginois, qui y entretenaient un grand feu signalant le port du large ?; et pour GB2, Lieu, selon toute vraisemblance ${ }_{2}^{8}$ de la plus ancienne installation de Carthage (qui disposait ainsi d'un port au N. de La Marsa, et d'un autre au S., Amilcar), Sidi-Bou-Saïd est rattaché à l'histoire ancienne et à l'aura qui l'entoure. Le village, dont le bleu des maisons est devenu le symbole de la Tunisie, résumerait en ses murs tout le passé du pays. De plus, la pratique du mal(o)uf le relierait à l'Andalousie arabe, symbole de tolérance et de culture.

31 Tout un syncrétisme, mêlant présent (à découvrir) et passé (à connaître), vus par des regards orientés, s'esquisse à partir de ces données. 


\subsection{La figure de Saint Louis} l'objet d'une légende, ignorée par GB1 [l'exposé «Histoire» qui précède le chapitre consacré à Tunis, précise En 1270, la ville fut attaquée sans succès par St Louis, qui mourut dans son camp de Carthage.], GV qui pose que C'est du sommet de cette colline que les guetteurs arabes virent approcher la flotte de la croisade dirigée par saint Louis (1270) et GG qui sélectionne le soufisme comme guide de visite et cite d'abondance le sociologue et ethnologue Jean Duvignaud, faisant de ces citations la justification et l'exemplification de son point de vue, ce qui le distingue des autres articles consacrés au village : Les Européens savent peu que ces ruelles montantes, ces villas closes, ces cafés paisibles sont le décor d'une mysticité fervente, celle de l'islam maghrébin pour lequel le culte des saints est fondamental. Sidibou-Saïd est le prototype du village maraboutique ${ }^{10}$.

\subsection{2. ... à la légende}

37 Ignorée de GB1, GV, GG et B (dont la rapidité des exposés, due au nombre de pages et au format de cette série, peut justifier de nombreux silences), elle est reprise et développée de GB2 (1997) à GN, GGT et GR (2002). On peut relever qu'elle y est explicitée en tant que telle, et que de nombreuses marques de subjectivité s'y lisent dans certains cas.

La confusion de saint Louis et Sidi Bou Saïd consacre le syncrétisme opéré par le discours touristique. Cette « légende », apparue récemment et fort peu connue localement, ré-écrit la fin de la vie de Louis IX, avec quelques variantes.

Ainsi, GB2 fait intervenir un marabout dont la sagesse le [saint Louis] séduisit. Celui-ci [le marabout] lui ayant suggéré d'abandonner croisade et royauté en faisant croire à sa mort... En revanche, l'intervention décisive, dans les quatre guides relatant la légende, est celle 
d'une superbe beauté berbère (GB2), syntagme reformulé en charmes du pays et d'une jeune berbère (GN), belle berbère (GR) et simplement une femme [dont il était tombé amoureux] (GGT), qui laisse ouverte l'appartenance de cette femme à un peuple et à une religion.

En revanche, les occupations du roi et le lieu de rencontre sont identiques : il faisait de longues promenades (GB2), GR renchérissant : il se promenait seul sur la plage...

41 Sagesse et amour s'unissant, le roi se convertit donc à l'islam et devient le saint patron du village : il s'initia au Coran et devint le saint personnage que l'on vénère. Sous le nom de Sidi Bou Saïd (GB2), il embrasse par la même occasion l'Islam, et devint Sidi-Bou-Saïd, ce saint vénéré aujourd'hui. (GR).

Quant à sa mort due à la peste, il s'agirait d'un stratagème pour lui permettre de vivre une seconde vie: un sosie, parfois un pauvre homme, opportunément atteint de la peste, succombe à sa place. Fusion du christianisme et de l'islam, le saint Louis de la légende pourrait ainsi se réclamer d'une double sainteté, et marquer de façon indélébile un lieu tunisien d'un sceau français.

$\mathrm{Si}$ GR, notamment, multiplie les marques de subjectivité : [une autre version], à laquelle nous adhérons bien sûr ${ }^{11}$, trop beau pour être vrai !, il n'en rapporte pas moins cette histoire en la reformulant et en la développant, les indices de subjectivité impliquant la distance entre le dire et le dit, et créant une complicité avec le lecteur - touriste averti. Mais par rapport à quoi et à qui y a-t-il prise de distance ?, des naïfs lecteurs?, des habitants de Sidi-bouSaïd, susceptibles de croire ?, du moins de raconter ? ...

Le village devient ainsi le creuset d'histoire et de civilisations communes, où Occident chrétien et Maghreb musulman s'unissent et se fondent. L'évocation de la Carthage punique se mêle à la tentative de siège de Tunis (pour les uns), de Carthage (pour les autres) par les croisés, leur échec répondant implicitement aux guerres puniques et à la victoire des Romains.

\section{Sidi-Bou-Saïd, village symbolique de la Tunisie par la grâce des Européens?}

45 A l'exception notoire de $\mathrm{GB} 1$ et $\mathrm{B}$, nombreux sont les écrivains évoqués, très majoritairement français, ainsi que quelques peintres. Ces listes ont valeur d'incitation à visiter le village, voire à boire un thé ou un café, ou encore à s'initier à la chicha, à leur exemple, au Café des Nattes.

Et que serait Sidi-Bou-Saïd sans le baron d'Erlanger? La présence d'illustres européens, artistes ou mécènes, justifie ainsi l'invasion touristique regrettée par les ouvrages mêmes qui poussent à voir, mais aussi contribue à façonner le pendant de Saint Tropez ou de Saint Paul de Vence: village posé comme symbolique de la Tunisie, Sidi-Bou-Saïd est digne de figurer parmi les hauts lieux touristiques français. Après avoir exalté la beauté du village, GGT s'interroge pourtant: Ce côté hautain [du village], on le ressent fortement lorsqu'on se promène dans le village; on se demande si le voyageur a bien sa place ici, à faire du tourisme, à acheter des souvenirs et à boire un verre. Mais la contradiction est vite levée : les souvenirs y sont souvent de meilleure qualité que ce que l'on trouve ailleurs et il s'agit presque d'une étape obligée. 


\subsection{D'illustres prédécesseurs}

47 Les XIX $^{e}$ et $\mathrm{XX}^{\mathrm{e}}$ siècles sont particulièrement sollicités; les passages de Chateaubriand, pour son Itinéraire de Paris à Jérusalem,ou de Flaubert pour sa Salammbô, sont cités, en relation implicite avec la vogue de l'Orientalisme. Mais c'est surtout le $\mathrm{XX}^{\mathrm{e}}$ siècle littéraire et pictural qui fait l'objet de nombreux rappels. Les noms cités sont dans leur très grande majorité, pour la littérature, ceux d'écrivains français; cette liste semble avoir valeur d'incitation forte à les imiter. GB1, GV et B se distinguent des autres guides par toute absence de références semblables. Ajoutons que l'« Aperçu religieux, artistique et littéraire » » de GB1 ne traite que des données artistiques spécifiques de l'Algérie et de la Tunisie, que GG cite abondamment Jean Duvignaud, pour l'étude du soufisme et des processions, et que GV signale que Sidi Dhrif, disciple du sage Abou Saï, fut l'« auteur de l'énumération des treize modes classiques du malouf ", dans son recueil Noria des modes, indiqué par GB2.

Le nom du XXe siècle le plus couramment cité (il figure dans tous les guides signalés, à l'exception, donc, de GB1 et B) est celui de Klee, sans doute en raison de ses tableaux de la période tunisienne ; Gide (GB2, GN, GGT), Bernanos (GB2, GGT, GR), Simone de Beauvoir (GG, GN, GR) sont suivis, dans ce tableau d'honneur, par Montherlant (GB2, GR) et Colette (GN, GR), Macke n'apparaissant, en compagnie de Klee, que dans GG, et Lamartine dans GR. La voix et les protestations de Julien Benda sont évoqués dans GG, seul à parler du peintre Jellal Ben Abdallah, et à relier les séjours de Klee, Macke, Gide, Bernanos à la personne du baron d'Erlanger. GB2, lui, va jusqu'à dire du Café des Nattes qu'il est « fameux entre tous car fréquenté autrefois par Gide, Bernanos, Klee, Montherlant ».

Cette manifestation d'un regard européen (et français) pose aussi, implicitement, la question: «fameux", pour qui? Pas pour les Tunisiens et moins encore pour les habitants de Sidi-Bou-Saïd, qui le fréquentent assidûment depuis de longues années. Ce guide pour touristes puise critères et valeurs à la source, celle des touristes, au risque d'un discours circulaire et fermé. Le sous-titre de GB2 Le Saint Paul de Vence tunisien appartient à un sous-ensemble discursif qui regroupe ces assertions : Il est de bon ton de comparer Sidi Bou Saïd à Saint Tropez ou à Saint-Paul-de-Vence (GV) ${ }^{12}$, Véritable image d'Epinal ... Sidi Bou Saïd est une sorte de Montmartre huppé ou de St-Paul-de-Vence (GN), Il est de bon ton de comparer «Sidi-Bou» à Saint-Paul-de-Vence ou à Saint-Tropez (GR) ${ }^{13}$. GG se contente d'un sous-titre, "Sidi-Bou-Saïd» à l'avant-garde de la Jet-Set, qui introduit un court exposé consacré au baron d'Erlanger. En raison de son (très) petit format, B s'abstient de tout développement et se contente de signaler: ses rues pavées sont aujourd'hui envahies par les cars touristiques.

50 C'est bien le passage et la fréquentation de ces lieux par d'illustres artistes européens qui en font l'importance et leur donnent sens. On le voit, seules quelques exceptions (GB1 et GG) se distinguent de cette célébration européenne à usage de touristes (français et européens ?), l'invasion touristique de ce village "incontournable » côtoyant dans les mêmes textes l'affirmation qu'il s'agit d'un village "maraboutique", habité par une «mysticité fervente»: raison supplémentaire pour s'y rendre et y re-créer d'autres villages de la Côte d'Azur? 


\subsection{Le (re-)créateur de Sidi-Bou-Saïd}

51 La figure du Baron d'Erlanger occupe une place importante dans la majorité des guides, à quelques exceptions significatives. Son nom ne figure pas dans GGT (dont l'édition de 2000 n'est que la ré-impression de celle de 1993) dans la partie consacrée aux arts, ni dans le chapitre traitant du malouf, ni dans celle traitant de l'architecture, ni dans l'article Sidi-Bou-Saïd. En revanche, la très longue première partie du livre signale l'opposition entre toits plats, héritage d'une antique civilisation urbaine arabe,et toits pointus, comme ceux des maisons de Sidi-Bou-Saïd, reflets de l'influence marocaine qui s'est fait sentir à partir $d u$ XIIIe siècle. L'article consacré au village serait ainsi mis en relation avec l'histoire religieuse et architecturale de la Tunisie. Peut-on aussi aller jusqu'à lire une allusion à l'action du baron d'Erlanger dans cet énoncé : Il n'y a pas d'endroit mieux préservé sur tout le littoral méditerranéen... ?

Même GB1 le cite, comme arabisant notoire, et indique l'importance de ses collections. Sa maison (ou palais), le dar Nejma ez Zohra figure dès GV comme l'une des visites à accomplir (palais...récemment restauré et qui abrite un intéressant musée des instruments de musique). Mais les indications de GV s'arrêtent là ; GG informe sur le baron et le rôle qu'il a joué dès 1912, indique rapidement : presque incidemment, au détour d'une rue, on pénétrera dans le palais $d u$ baron d'Erlanger, devenu récemment un musée de la Musique méditerranéenne dédié - notamment - à la gloire du malouf, et ne parle de ce palais que par une citation d'Ahmed Karoui, présentant son architecture et sa décoration. Il se distingue, par cette sobriété, des autres guides ; le silence de B l'oppose à tous.

En revanche, la présentation de l'homme, de sa demeure et de son rôle est longuement évoquée dans l'autre sous-groupe, constitué de GB2, GN et GR.

\subsubsection{La figure du baron d'Erlanger}

Présente dans GB1, sa qualité d'arabisant (notoire) disparaît au profit de sa vie et de son œuvre, architecturale et musicale.

GB2 développe le rôle qu'a joué à Sidi-Bou-Saïd et en Tunisie le fait qu'il s'y soit installé : Le village doit son visage actuel à la venue, en 1912, du baron Rodolphe d'Erlanger ${ }^{14}$ (1872-1932). Le même guide rappelle, comme fondements de ce jugement, les actions qu'il a entreprises : décret de protection intégrale du site (1915), revalorisation de l'architecture tunisienne traditionnelle, réanimation de certaines formes de l'artisanat, ajoutant: C'est grâce à lui, sans doute $e^{15}$, que Sidi-Bou-Saïd devint, et demeure un lieu fréquenté par les écrivains, les artistes, les musiciens et les poètes. En témoigne l'ouverture du Centre des musiques arabes et méditerranéennes dans sa demeure.

GN lui consacre tout un paragraphe reprenant l'essentiel de ces données, et précise qu'il débuta comme peintre orientaliste, s'éprit de la musique arabe dont il devint un grand expert ... et qu'il fut même choisi par le roi Fouad Ier d'Egypte pour préparer le grand congrès du Caire consacré à cette musique. Même le bicolorisme bleu et blanc serait son fait. Ce dernier point est repris par GR : Il [...] choisit d'imposer une couleur unique pour les boiseries des portes et des fenêtres. Ce bleu lumineux devenu aujourd'hui la couleur fétiche et le symbole de la Tunisie fut donc inventé ${ }^{16}$ par un aristocrate britannique.

Ainsi donc, le baron d'Erlanger ne fut pas seulement un mécène : c'est à lui que village et symbolisme devraient leur véritable naissance et leur essence. 


\subsubsection{Le dar Nejma ez Zohra, palais (à visiter) du baron d'Erlanger}

\section{résume et subsume le charme, le secret et la beauté de Sidi-bou-Saïd. Il s'agit donc ici de} rendre un hommage insistant à celui qui aurait su (re)créer l'essence même de l'art et de la vie "mauresques ${ }^{17}$. Selon les points de vue adoptés par chaque ouvrage s'étagent ainsi le (bref) rappel du baron, la célébration de sa demeure, et le rôle qu'on lui prête dans le renouveau artistique. On en comprend mieux le quasi silence de GV et de GGT, voire de GG citant en forme de sous-titre Ahmed Karoui : «Sidi-Bou-Saïd, vu d'en haut, ressemble à un disque blanc où se confondent le bleu foncé de la mer et celui, plus clair, lui venant du ciel ».

61 Ainsi exaltée, décrite et commentée, l'œuvre du baron d'Erlanger est la quintessence de ce village maraboutique, l'un des plus beaux « du rivage méditerranéen ». De l'amateur éclairé de l'art musulman, du musicologue et du « décideur » au démiurge suggéré par les guides, la distance est sans doute grande...

\section{Un village tunisien à usage de touristes « avertis »?}

Les différents lectorats visés par les guides retenus peuvent être plus ou moins sensibles à tel ou tel aspect retenu. Ainsi, «boire un verre "au Sénéquier du coin, à savoir le café des Nattes, semble un argument pour les « Routards », qui risquerait de déplaire aux touristes visés par GGT. Plusieurs doxas se construisent donc dans ce corpus discursif, en fonction des visées particulières. Mais le discours de doxa n'en constitue pas moins une caractéristique du corpus.

De même, le jeu sur le connu et le nouveau, à découvrir et mémoriser, se retrouve dans chaque guide : l'histoire antique, celle des croisades, plus près de nous celle des $\mathrm{XIX}^{\mathrm{e}}$ et $\mathrm{XX}^{\mathrm{e}}$ siècles ${ }^{18}$, histoire générale et histoire particulière mêlées; on l'a vu, tout un sousensemble ne recule pas à faire état de la supposée conversion de Louis IX à l'Islam ... La mémoire discursive s'y lit à plein, relayée par une mémoire discursive plus proche, ravivée par les noms de "grands hommes", "découvreurs» de ces lieux, et, éventuellement par les titres de leurs œuvres.

Ces différentes données, peu ou prou présentes et diversifiées, construisent donc un syncrétisme de plus ou moins bon aloi, tendant à confondre des traditions distinctes et 
des histoires vécues autrement, dans un ensemble où tous, Tunisiens et touristes étrangers, pourraient trouver leur place, en confondant leur commun « éblouissement ».

Des spécificités se dessinent pourtant, justifiant l'existence de nombreuses collections, éventuellement dans une même maison d'édition. Ainsi, la célébration du baron d'Erlanger, ou le long exposé sur le soufisme, appuyés sur des discours " autres ${ }^{19}$ qui font office de discours d'autorité surplombant et constitutif de la doxa, points de vue différents donnant sens et orientations différentes de visites, n'en participent pas moins à ce discours touristique général. Le discours autre est ainsi évoqué et masqué, par le lissage opéré par l'énonciation du guide.

66 PETIOT, G. (à par. 2002) « Autonymie et langue de bois », communication au Colloque «Le fait autonymique». Consultable sur le site: http://www.cavi.univ-paris3.fr/ ilpga/autonymie/actes.htm

67 REY-DEBOVE, J. (1978) Le métalangage, Paris : Editions Le Robert.

68 TOMASSONE R. (dir.) (2001), Une langue, le français, Paris : Hachette.

69 GUIDE BLEU Algérie Tunisie (1955) = GB1

70 GUIDE VISA En Tunisie (1996) $=$ GV

71 (Même texte in Guides Bleus Evasion Tunisie (1996-98)

72 GUIDE BLEU Tunisie (1997) = GB2

$73 \quad$ BERLITZ Tunisie guide de voyage (1998) $=\mathrm{B}$

74 GUIDE GALLIMARD Tunisie (1999) = GG

75 GUIDE NEOS Tunisie, $(2000)=$ GN

76 LE GRAND GUIDE DE LA TUNISIE Gallimard (2000) = GGT

77 GUIDE DU ROUTARD Tunisie (2002) = GR

\section{BIBLIOGRAPHIE}

Corpus de guides

Algérie Tunisie, Les Guides Bleus, Paris : Hachette, 1955.

En Tunisie, Guides Visa, Paris : Hachette, 1996.

Le grand guide de la Tunisie, Paris : Gallimard, 2000.

Tunisie, Guides Bleus Evasion, Hachette, Paris, 1996.

Tunisie, Guides Bleus, Paris : Hachette, 1997.

Tunisie, Guide de voyage, Paris : Berlitz, 1998.

Tunisie, Guide Gallimard, Paris : Gallimard, 1999.

Tunisie, Guide Neos, Paris : Michelin, 2000.

Tunisie, Le guide du Routard, Paris : Hachette, 2001. 


\section{Ouvrages}

AUTHIER-REVUZ, J. (1995) Ces mots qui ne vont pas de soi Boucles réflexives et non coïncidence du dire, 2 vol., Paris : Larousse.

AUTHIER-REVUZ, J. (2001) « Le discours rapporté », in Une langue, le français R. Tomassone (dir.), Paris : Hachette 2001, (192-201).

COURTINE, J.-J. (1981) Analyse du discours politique, Langages nº2.

COURTINE, J.-J. (1994) « Le tissu de la mémoire : quelques perspectives de travail historique dans les sciences du langage », Langages $n^{\circ} 114$, Mémoire, histoire, langage (J.-J. Courtine édit.).

DUBY, G. (1970) Histoire de la France, Paris : Larousse.

LE GOFF, J. (1966) Saint Louis, Paris : Gallimard.

PECHEUX, M. (1990) L'Inquiétude du discours, D. Maldidier édit., Paris : Editions des Cendres.

PERRET, M. (1994) L'Enonciation en grammaire de texte, paris : Nathan Université, 128.

\section{ANNEXES}

Corpus

TUNIS

Histoire [...] En 1270, la ville fut attaquée sans succès par St Louis, qui mourut dans son camp de Carthage. (p.526)

En face de la basilique [Saint-Cyprien], la grande route tourne à g. et passe sous la voie ferrée ; plus loin, on laisse en face la route de La Marsa pour prendre à dr. Et traverser la voie près de la gare de Sidi Bou Saïd. Au-delà, en appuyant à dr., on passe en vue du Dar Sidi Naceur Bey et on longe les jardins dits *Nejma Zohra, construits et aménagés au flanc de la colline, dans un site magnifique par feu le baron R. d'Erlanger.

Le baron d'Erlanger a réalisé là une œuvre architecturale de haut goût à laquelle ont été conviés les artisans indigènes de l'Afrique du Nord les plus réputés. Arabisant notoire, il a traduit les ouvrages anciens de la musique arabe, entre autres Al Farabi, et fondé un conservatoire pour la rénovation de l'art musical tunisien. Une partie de ses collections forme le musée d'Erlanger, installé à Dar Es Salam, rue principale à Sidi Bou Saïd.

20 k. Sidi Bou Saïd (hôt. Dar Nouar, près de la station, 6 ch., rest., tél. 29 ; - rest.- salon de thé Dar Zarrouk, du nom d'un ancien palais beylical, jardin, terrasse, *vue), village étagé dans un site admirable sur le flanc S.-O. d'un éperon de $130 \mathrm{~m}$. qui se termine au cap Carthage. La rue principale (sens unique), montant entre de jolies maisons mauresques, aboutit en face de la mosquée précédée de degrés inégaux et d'une galerie en bois (café maure et magasin de vente et exposition d'art artisanal tunisien sous le contrôle de l'U.S.C.A.T.) ; en arrière se dresse un élégant minaret. En contournant la mosquée à dr., on arrive près du Dar Zarrouk (V. ci-dessus) à dr.

A l'extrémité N. du village, phare-signal (portée : 24 milles) du sommet, *panorama magnifique.. Près du phare, cimetière musulman et koubba de Sidi Djebel. - Au-dessous, des falaises d'un rouge vif plongent vers la mer que borde une petite plage.

\section{L'EPOPEE TUNISIENNE}


[...] Sous cette dynastie [des Hafsides], le pays connaît une nouvelle période de prospérité et de paix malgré les difficultés : la croisade de saint louis, qui coûte la vie au ùonarque chrétien (1270), [...]. (p.51))

\section{SIDI BOU SAÏD **}

Août. Fête de la Kharja. Fête à caractère religieux.

Avec ses maisons chaulées de blanc, ses portails bleus sur lesquels de gros clous forgés dessinent des motifs géométriques, ses moucharabiehs finement ouvragés, ses jardins croulant de fleurs d'où s'échappent de hauts cyprès, Sidi bou Saïd incarne à la perfection le village de charme. Ce joyau de l'architecture traditionnelle est perché au sommet d'une colline qui servit de point de repère aux premiers navigateurs. Il offre des ruelles étroites et pentues, des maisons secrètement refermées sur des jardins, des vues sur des terrasses et des dômes qui se découpent sur le vert sombre de la végétation ou sur le bleu changeant de la mer. Le soir tombe, des odeurs subtilement sucrées de jasmin et de chèvrefeuille embaument l'air. depuis le succès rencontré par le café des Nattes, lieu de rendez-vous de la jeune bourgeoisie tunisoise et d'une clientèle internationale de passage, il est de bon ton de comparer Sidi Bou Saïd à Saint-Tropez ou à Saint-Paul-de-Vence. Comparaison assez réaliste durant les mois d'été ; le reste de l'année, Sidi Bou Saïd révèle son vrai visage, calme et paisible. La rue principale monte entre des boutiques de souvenirs où trônent les belles volières bleues et blanches, spécialité locale, vers l'escalier monumental du café des Nattes. Mais les rues avoisinantes restent le terrain de jeux des chats demi-sauvages et des enfants.

Un phare a remplacé aujourd'hui les feux-signaux qui guidaient les galères puniques vers le port. On peut monter sur la galerie du phare, après en avoir demandé la permission au gardien (prévoir rétribution). C'est du sommet de cette colline que les guetteurs arabes virent approcher la flotte de la croisade dirigée par saint Louis (1270). Un monastère fortifié y défendait la côte. Ce ribat, haut lieu spirituel et bastion du soufisme, une secte mystique d'Orient, fut la résidence d'Abou Saïd, pieux personnage d'origine marocaine, qui y vécut en ermite et attira de nombreux disciples. Plus tard, les corsaires devaient faire de Sidi bou Saïd le patron de la piraterie anti-chrétienne. Jusqu'en 1820, il fut défendu aux chrétiens de pénétrer dans ce village maraboutique.

Le sage Abou Saïd repose dans le haut de la ville, dans un marabout à quatre dômes orné d'un élégant minaret. Ses disciples sont enterrés dans un minuscule cimetière-belvédère ; parmi les tombes se trouve celle de Sidi Dhrif ( «le Gracieux »), auteur de l'énumération des treize modes classiques du malouf. Parmi les palais, trois sont ouverts au public. Le plus beau d'entre eux est le dar Nejma ez Zohra, palais du baron d'Erlanger récemment restauré et qui abrite un intéressant musée des instruments de musique (ouv. T.l.j. de 9h à $12 \mathrm{~h}$ et de 14h à 17h. Entrée payante). Le Dar Saïd a été transformé en hôtel, et le Dar

Zarrouk accueille un restaurant caché dans un jardin odorant et doté d'une terrasse à la vue imprenable (voir plus bas). Le soir, des banquettes de pierre du café Chergui, on peut assister à des concerts de musique malouf.

\section{Bonnes adresses (car. bleus)}

*** Sidi Bou Saïd, av. Sidi Dhrif [...] Un établissement confortable, affilié à l'école hôtelière, dont les élèves assurent le service. Jolie décoration intérieure de style oriental.

** Dar Saïd, rue Ettoumi [...] Installé dans un ancien palais s'ouvrant sur un patio au cœur d'un jardin rempli de fleurs, cet hôtel au confort simple a un charme particulier.

*Sidi Bufarès, 15 , rrue Sidi Boufarès [...] très bien situé, tout près du célèbre café des Nattes. Un tout petit hôtel au confort très simple mais propre, aménagé dans une maison 
traditionnelle.

*** Dar Zarrouk [...] Non loin du café des Nattes, sa terrasse offre une vue magnifique sur la mer. Cuisine un peu décevante. En haute saison, mieux vaut réserver. [...]

Des origines au protectorat

[...] L'attaque des croisés, en 1270, à laquelle la mort de saint Louis, à Carthage, mit rapidement un terme, ne fut qu'un orage sans lendemain. (p.69)

\section{Sidi Bou Saï***}

Sidi Bou Saïd est un ancien village maraboutique dont les maisons et les palais, étagés sur les flancs du jebel Manar - la «montagne du Phare ", un éperon qui domine la mer d'env. 130m. au cap Carthage -, composent un tableau admirable.

Quel sortilège en ce lieu, saint pour les musulmans, incite-t-il tout un chacun à n'y errer qu'à pas lents et feutrés, indifférents à l'agitation de ses rues au cœur de l'été, voire oublieux de nos réflexes photographiques alors même qu'on a l'appareil à la main? C'est le charme tranquille de Sidi Bou Saïd, de ses ruelles pavées, de ses jardins secrets et patios ombreux invisibles de l'extérieur, de ses façades immaculées dont la blancheur s'enrichit de linteaux et jambages sculptés, de fenêtres à moucharabieh, de toits de tuiles vernissées, puis s'apaise dans le bleu - l'incomparable «bleu Sidi Bou Saïd » - de grilles renflées et de portes à clous noirs et se renouvelle dans le jeu des volumes.

C'est, le soir venu, le silence parfumé par la plainte d'une musique de malouf. C'est, dans nos souvenirs, celui des descriptions lyriques de Gide ou des couleurs de Klee. C'est la légende de saint Louis séduit avant nous...

Visite : Si vous arrivez en voiture, prenez la rue qui serpente au flanc du cap Carthage et vous atteindrez un parking où vous devez obligatoirement laisser votre véhicule (plan Carthage C1).

Le palais du baron d'Erlanger est tout près du parc de stationnement. On ne visite pas Sidi Bou Saïd, on s'y promène un peu au hasard. Ca et là, des portes entrouvertes vous permettent de glisser un regard furtif sur des courettes ensoleillées, aux murs ornés de rafraîchissants zelliges (carreaux de céramique).

\section{De la légende ...}

On raconte que, las d'un siège qui s'annonçait mal, son armée décimée par la peste, le saint roi, venu sur la terre d'Afrique pour « chrestienner le roi de Thunes et son peuple ", faisait de longues promenades solitaires et rencontra un jour un marabout dont la sagesse le séduisit. Celui-ci lui ayant suggéré d'abandonner croisade et royauté en faisant croire à sa mort - il y avait justement à Tunis un parfait sosie de lui et il se mourait de la peste -, le roi n'abandonna tout scrupule qu'au passage d'une superbe beauté berbère. L'ayant épousée, il s'initia au Coran et devint le saint personnage que l'on vénère. Sous le nom de Sidi Bou Saïd.

\section{... à l'histoire}

Lieu, selon toute vraisemblance, de la plus ancienne installation de Carthage (qui disposait ainsi d'un port au N. La Marsa, et d'un autre au S., Amilcar), le promontoire de Sidi Bou SaId fut pourvu, après la conquête arabe, d'un ribat faisant partie d'une chaîne de forts qui défendaient le rivage du golfe de Tunis. Les Espagnols s'en emparèrent en 1535 et n'en furent chassés par les Turcs qu'en 1574.

\section{Un foyer religieux}

C'est vers 1207, au retour d'un pèlerinage à La Mecque, que s'installe sur le cap Carthage un mystique nommé Abou Saïd Khalafa ben Yahia el Temimi el Beji. Pendant près de vingt 
ans, il oeuvra pour la diffusion du soufisme, suscitant l'admiration par ses pratiques ascétiques, et sa mort, en 1236, fit de lui le saint patron du village (qui ne prit cependant son nom qu'au XIXe siècle). Ses disciples continuèrent son action, drainant vers son tombeau l'hommage des confréries religieuses et faisant de la colline un haut lieu de spiritualité.

\section{Le «Saint-Paul de Vence» tunisien}

A partir du XVIIIes., princes, ministres, hauts fonctionnaires et notables font du jebel Manar leur lieu de séjour favori pendant la saison estivale et s'y font construire palais et résidences d'été.

Le village doit son visage actuel à la venue, en 1912, du baron Rodolphe d'Erlanger (1872-1932) ; c'est à son instigation qu'est promulgué en 1915 un décret de protection intégrale du site qui constituait à l'époque - et pour le monde entier - une innovation ; c'est lui qui, se portant acquéreur de palais, les fait restaurer pour prêcher l'exemple, revaloriser l'architecture tunisienne traditionnelle et réanimer certaines formes de l'artisanat. C'est grâce à lui, sans doute, que Sidi Bou Saïd devint, et demeure un lieu fréquenté par les écrivains, les artistes, les musiciens et les poètes. En témoigne l'ouverture du Centre des musiques arabes et méditerranéennes dans sa demeure. * En haut de la ville, en arrière du CAFE DES NATTES, se trouve la mosquée, près d'une zaouïa ; sans doute fondée peu après la mort d'Abou SaId, elle fut restaurée par les Husaïnides auxquels on doit l'élégant minaret de la zaouïa et plusieurs fontaines.. $\checkmark$ LE CIMETIERE renferme les tombes de plusieurs mystiques, disciples d'Abou Saïd, dont l'un, dit Sidi Dhrif (« le Gracieux ») est l'auteur de la « Noria des modes », énumération des treize modes classiques du malouf. De là, très belle vue sur le golfe de Tunis. a Parmi les palais, quelques-uns sont accessibles au public :

NEJMA EZZAHRA **, le palais du baron d'Erlanger, construit en 1921 par des artisans marocains et tunisiens se trouve dans un magnifique jardin aux essences odoriférantes. Cette maison, avec sa longue façade blanche, ses moucharabiehs bleus, ne se découvre pas de l'extérieur - caractéristique de l'architecture du Maghreb.

A l'intérieur, en revanche, on admirera la splendeur du décor : plafonds de bois peints et dorés, colonnes ciselées, pavements de marbre, le musée d'instruments de musique, et la riche collection de manuscrits arabes, de verres de Venise et de Turquie, d'objets en argent et en bois incrustés de nacre, etc.

DAR SAÏD est devenu un hôtel, et DAR ZARROUK abrite un restaurant d'où la vue est splendide.

En marge gauche, p. 160 : dessin (en haut) : maison à moucharabiehs et fenêtres grillagées

notes : (caractères couleur bleue)

En haut de la ville, vous pourrez boire un thé à la menthe au café des Nattes. Fameux entre tous, car il était fréquenté, autrefois, par Gide, Bernanos, Klee, Montherlant... La spécialité de Sidi Bou Saïd : les volières en fil de fer, bleues et blanches.

\section{En caractères noirs :}

La musique maluf, composée de pièces vocales - en solo ou en chœur - et instrumentales avec un petit orchestre (luth, timbales, flûte, rebec, et psaltérion, provient d'Andalousie ; elle fut introduite en Tunisie dès le XIIe s. Alors que cette musique raffinée semblait en voie de disparition, le baron d'Erlanger l'a relancée au début du siècle, et l'on peut aujourd'hui entendre encore des concerts de maluf, notamment lors de la fête de la Kharja à Sidi Bou Saïd. (p.159-161) 


\section{Sidi Bou Saïd}

Entre le phare qui coiffe sa colline et le bord de mer, Sidi Bou Saïd présente un décor type de carte postale. Construit en cascade sur une pente raide, il présente des maisons cubiques immaculées aux volets et portes bleus. Le village est célèbre pour son incomparable beauté. Du sommet de la colline, vous contemplerez l'un des plus beaux panoramas qui soient. De plus, vous pourrez admirer les fameuses cages à oiseaux de la région, dont les arabesques de bois et de fils de fer rappellent les élégantes courbes en fer forgé des fenêtres des maisons.

Le village soit son nom à la mosquée et à la tombe d'un saint homme du XIIIe siècle. Devenu, il a une centaine d'années, la retraite exclusive de riches personnages locaux, ses rues pavées sont aujourd'hui envahies par les cars touristiques. Afin de profiter au mieux de votre séjour, allez-y le soir ou tôt le matin, l'idéal étant de passer une nuit dans l'un des beaux hôtels locaux. Deux cafés sont renommés : le café des Nattes et le café Chaabane ; tous deux offriront une vue enchanteresse sur la mer et le village. (p. 39)

\section{Sidi-Bou-Saïd (p.128)}

Le plus pur, le plus beau village tunisien est certainement Sidi-Bou-Saïd. Les Européens savent peu que ces ruelles montantes, ces villas closes, ces cafés paisibles sont le décor d'une mysticité fervente, celle de l'islam maghrébin pour lequel le culte des saints est fondamental. Sidi-bou-Saïd est le prototype du village dit maraboutique, car fondé par sidi (saint) Bou Saïd. Le lieu semble chargé de densité religieuse : celle-ci remonte-t-elle aux Carthaginois, qui y entretenaient un grand feu signalant le port du large ? A sidi Mahrez, saint patron de Tunis, qui s'y retirait? A l'ermitage qu'y fonda Sidi Bou Saïd ? «le foyerr [du village] est en haut de la colline : le marabout qui contient les restes du saint [...], explique Jean Duvignaud. Le soir, quand les croyants s'assemblent et se prosternent, la découpe des terrasses et des dômes dessine une architecture simple et abstraite [...] » Une procession. « Les groupes de dévots, les uns à reculons, les autres avançant lentement, se répondaient en chantant et en s'inclinant d'avant en arrière, selon le mouvement qui, cent fois répété, engendre la transe, voire l'extase. la procession monta [...] jusqu'au café des Nattes, poursuivant son dialogue psalmodié, accompagné du youyou des femmes, derrière les moucharabiehs » (Jean Duvignaud). Ces confréries soufiques associent à la méditation et aux techniques de transe la musique andalouse, qui possède ici son musée.

\section{(Marge gauche) « SiIDI-BOU-SAÏDdi-Bou » A L'AVANT-GARDE DE LA JET-SET}

En 1912, le baron Rodolph Erlanger (1872-1932), sujet britannique, tomba amoureux du village maraboutique. Celui-ci est, depuis le XVIIIe siècle, le haut lieu du Tout-Tunis : les notables, les ministres, le bey y ont leurs résidences. Le baron fit classer le site en 1915, s'y construisit un palais, restaura les demeures anciennes. Klee, Macke, Gide, Bernanos, entre autres, y séjourneront.

\section{LA MODE DES BAINS DE MER}

Les élites beylicales ne pouvaient ignorer la mode des bains de mer qui gagna l'Europe au XIXe siècle. Mais elles l'adaptèrent aux mœurs locales, en faisant édifier les maâouma, baraques en bois perchées sur des pilotis au-dessus des flots, dont le plancher était percé pour permettre aux dames d'entrer dans l'eau directement et de se baigner sans être vues.

Illustrations, photos : café des Nattes, Baron d'Erlanger, représentation d'unes barques au bord de l'eau. 
(p.199) : photos de Sidi-Bou-Saïd, et, marge droite (commentaires de photos) : QUAND SIDI-BOU-SAÏD INSPIRRE LES ARTISTES

«J'imagine... J'imagine... » glapit de sa voix de fausset Julien Benda qui, transporté à Sidibou-Saïd par Simone de Beauvoir, refuse de descendre de l'auto. A cette seule exception, les bleus et les blancs du village mythique ont conquis et conquièrent encore des générations d'artistes. Macke et Klee furent de ceux-là. Klee rapporte : « Sidi-Bou-Saïd, le dos d'une montagne sur laquelle on voit poindre, selon un rythme rigoureux, les blanches formes des maisons. » Désormais, l'éblouissement de Sidi-Bou-Saïd va transfigurer sa peinture. Le peintre Jellal ben Abdallah y vit est son atelier surplombe la mer. On y trouve de nombreuses galeries d'art contemporain. Presque incidemment, au détour d'une rue, on pénétrera dans le palais du baron d'Erlanger, devenu récemment un musée de la Musique méditerranéenne dédié - notamment - la gloire du malouf. Ahmed Karoui présente ainsi ce lieu : «l'édifice, concçu et construit dans la tradition tunisienne, est d'une grande beauté. L'intérieur est orné [...] de sculptures sur stuc et de galeries revêtues de céramique. [Le palais] est pourvu également d'une magnifique piscine ombragée de cyprès. » (Ci-contre, photos prises dans les ruelles du village.)

Sidi Bou Saïd (caractères bleus)

Comptez 1 h. [...]

Véritable image d'Epinal de la Tunisie éternelle, avec ses maisons aux murs d'une blancheur éclatante ponctuée de volets et de moucharabiehs bleus, ses ruelles pavées et pentues, Sidi Bou Saïd est une sorte de Montmartre huppé ou de St-Paul-de-Vence. Perché sur une colline, ce charmant village surplombe Carthage et le golfe de Tunis. L'absence d'autos favorise une ambiance joyeuse et feutrée que renforcent les fières maisons de Sidi Bou Saïd. Chacune laisse deviner un mystère qui, hélas, reste entier. Aucune de ces demeures cossues ne peut être visitée, hormis le palais du baron d'Erlanger. On peut cependant rêver devant les lourdes portes cloutées qui s'ouvrent parfois sur de somptueux jardins odorants et touffus, remplis de bougainvillées, de chèvrefeuilles et de vignes sauvages. Gide, Colette, Simone de Beauvoir, Paul Klee et bien d'autres artistes sont venus ici mettre leurs pas dans ceux de Flaubert et de Chateaubriand.

\section{En flânant dans Sidi Bou (car. bleus)}

Laissez-vous guider le long de la rue principale qui vous conduira jusqu'aux imposantes marches du célèbre café des Nattes*. [en car. bleus] On ne peut que succomber au rituel du thé à la menthe servi avec des pignons, assis en tailleur au milieu des fumeurs de chicha. En continuant l'ascension vers le sommet du village, en direction du phare, on rencontre un petit cimetière aux pierres tombales blanches légèrement incurvées sur le dessus. Ces creux qui gardent l'eau de pluie sont appelés « bols des oiseaux ». En redescendant un peu la rue, on atteint le phare situé juste en dessous du cimetière ; ce n'est pas un monument touristique mais les rares curieux sont bien accueillis. Du haut de cette petite tourelle rouge, le regard embrasse la baie bleutée, fermée au loin par une majestueuse montagne souvent baignée de brume, le mont Bou Kornine.

Pour le reste, Sidi Bou Saïd se respire davantage qu'il ne se visite. Il faut s'éloigner du centre pour découvrir des ruelles un peu moins retapées et aseptisées à l'intention des touristes et des étrangers à demeure. En redescendant du phare, rue Taïeb Méhiri, on aperçoit les coupoles de la zaouïa où repose Abou Saïd, le saint patron du village. Une légende voudrait que ce personnage fût en réalité... Saint Louis. Ayant succombé aux charmes du pays et d'une jeune Berbère, le roi pieux aurait pris l'identité d'Abou Saïd, 
tandis qu'un pauvre homme, opportunément son sosie, se mourait de la peste ! la réalité est plus prosaïque : Saint Louis est bel et bien mort de la terrible maladie et Sidi Bou Saïd, mystique d'origine marocaine, fut pour sa part une figure du soufisme. Ce lieu de recueillement est malheureusement fermé aux non musulmans.

L'entrée du palais du Baron d'Erlanger est rue du 2-Mars 1934, en bas du village près du parking. Ennejma Ezzahra**, [car. bleus] le palais que se fit construire le baron d'Erlanger et qu'il ne cessa d'arranger entre 1912 et 1922, est devenu le Centre des musiques arabes et méditerranéennes ( $9 h-13 h / 14 h-17 h$ en hiver, $9 h-12 h 30 / 15 h-18 h 30$ en été, ferméle lundi ; entrée payante). Il abrite de multiples activités liées à la conservation et à la diffusion du patrimoine musical. Des concerts y sont régulièrement organisés. On y accède par une allée d'aloès et de petits orangers. Entouré d'un grand parc dominant la mer, agrémenté de jardins andalou et persan, le palais dispose de superbes salles riches en colonnes et en panneaux de bois ajouré. Le salon de musique, mla bibliothèque, la fontaine en marbre noir du grand salon sont un véritable émerveillement. Tous ces lieux retrouvent leur véritable vocation lorsque les cordes du luth vibrent d'une pièce à l'autre, comme au temps du baron.

Le Baron d'Erlanger (1872-1932) [car. bleus]

Héritier de riches banquiers français d'origine allemande, mais élevé à Londres, le baron Rodolphe d'Erlanger s'établit à Sidi Bou Saïd en 1912. Il espérait renforcer dans ces lieux enchanteurs une santé réputée fragile. Il y donna libre cours à sa double passion de la peinture et de la musique, tout en manifestant le désir presque obsessionnel de figer ce merveilleux village dans un bicolorisme bleu et blanc. En 1915, il obtint des autorités coloniales que le site fût protégé par décret. Le baron, qui débuta comme peintre orientaliste, s'éprit aussi de la musique arabe dont il devint un grand expert, au point de lui consacrer un traité en six volumes parus entre 1930 et 1959 à Paris, aux éditions Paul Geuthner : « La Musique arabe, ses règles, leur histoire. » En 1932, il fut même choisi par le roi Fouad 1er d'Egypte pour préparer le grand congrès du Caire consacré à cette musique.

\section{HISTOIRE ET SOCIETE}

\section{[...] Les chrétiens}

Le pays paraissait si civilisé et gouverné avec tant de sagesse que le pape Innocent IV exclut même toute possibilité de croisade. Mais le roi de France Louis IX, le futur Saint Louis, peut-être autant pour des raisonscommerciales que religieuses (le bruit courait que l'émir était désireux de se convertir) débarqua à Carthage le 18 juillet 1270 avec ses croisés (ce fut la septième et dernière crroisade). [...)

Tunis fut assiégée pendant un mois et al-Mustansir était sur le point de battre en retraite lorsque Saint Louis mourut victime de la peste. [...] p.45-46)

\section{Sidi-Bou-Saïd}

Le village perché de Sidi-Bou-Saïd (à $2 \mathrm{~km}$ ) se dresse à l'extrémité de la route de Carthage. Avec ses maisons étonnantes de blancheur et son site remarquable, il s'agit d'une excursion agréable après la visite parfois un peu fastidieuse des ruines carthaginoises, qu'il semble dominer avec un certain dédain.

Ce côté un peu hautain, on le ressent fortement lorsqu'on se promène dans le village ; on se demande si le voyageur a bien sa place ici, à faire du tourisme, à acheter des souvenirs (souvent de meilleure qualité que ce qu'on trouve ailleurs) et à boire un verre - il s'agit presque d'une étape obligée - à la terrasse du café des Nattes. On déambule au hasard des ruelles et des allées qui gardent leurs secrets derrière leurs volets clos; on se laisse 
éblouir par la blancheur des murs et par le bleu des portes cloutées et des fenêtres à moucharabieh (balcon fermé par un grillage), avec, çà et là, la mer qu'on entrevoit. Sidi-Bou-Saïd, comme Mdina à Malte et quelques autres villages méditerranéens, semble ignorer les touristes. Les habitants savent se garder des regards indiscrets et considèrent l'arrivée des touristes comme une invasion passagère. Lorsque les touristes sont repartis, le village retrouve sa tranquille existence, et seuls les chats errent dans les rues. Sidi-Bou-Saïd doit son nom à un saint du XIIIe siècle dont la tombe et la zaouia furent construites sur les site d'un ancien ribat arabe et d'un phare. La légende veut que Saint Louis, lors du pillage de Carthage, se soit réfugié en ce lieu avec une femme dont il s'était épris. Les Espagnols occupèrent la ville au XVIe siècle, de 1535 à 1574. Leurs successeurs, des musulmans chassés d'Andalousie (dont certains qui pratiquaient la piraterie firent de Sidi-Bou-Saïd leur protecteur), indignés par les mœurs dissolues des infidèles, interdirent l'accès du village à tous les non-musulmans jusqu'en 1819.

Sidi-Bou-SaId, qui s'est développé plutôt lentement depuis le début du siècle, est devenu un lieu fréquenté par de nombreux artistes et écrivains européens : André Gide, Georges Bernanos, le peintre Paul Klee. Il n'y a pas d'endroit mieux préservé sur tout le littoral méditerranéen, et il fait bon s'y promener. Il est possible de séjourner à l'hôtel Sidi-BouSaïd, magnifiquement situé [...]

\section{SIDI-BOU-SAÏD}

« Où l'on entre dans une ruelle, comme au couvent. A pied humble, dans l'odeur du jasmin. » Un poète...

Il est de bon ton de comparer « Sidi-Bou » à Saint-Paul-de-Vence ou à Saint-Tropez. C'est un jugement un peu hâtif, autant dire faux. Si Sidi-Bou-Saïd est effectivement très touristique et hyper fréquenté en été, on se contente d'y passer seulement quelques heures mais qui valent vraiment le coup. L'infrastructure hôtelière, assez réduite, ne permet pas de séjour prolongé. Sidi-Bou-Saïd, accroché au flanc du djebel Manâr (la montagne du Phare) qui veille sur le golfe de Tunis, est un véritable balcon sur la mer. «Perle blanche suspendue au-dessus du golfe d'azur », comme l'écrivent les brochures touristiques. Mais on pourrait aussi utiliser bien d'autres métaphores pour décrire le plus fameux des villages tunisiens où se mêlent, avec un rare bonheur, des éléments architecturaux des Cyclades et de l'Andalousie. Le blanc et le bleu se complètent dans une harmonie qui célèbre ici les «noces du ciel et de la mer ».

Chateaubriand en route pour Jérusalem, Flaubert à la recherche de Salammbô et Lamartine avaient découvert le golfe de Tunis, de ce promontoire naturel d'où partaient les signaux guidant les galères puniques vers le port. Plus près de nous, Bernanos, Gide, Colette, Simone de Beauvoir et Montherlant (excusez du peu) trouvèrent refuge ici. Michel Foucault y écrivit notamment L'Archéologie du savoir. Quant au peintre Paul Klee, il tomba sous le charme de cette architecture au point d'enfermer dans les coupoles de peits cubes de couleur, comme des oiseaux en cage.

\section{UN PEU D'HISTOIRE}

En 1912, un baron anglais, Rodolph d'Erlanger (1872-1932), découvre Sidibou, décide de s'y faire construire une somptueuse demeure et de restaurer les anciennes maisons. Il obtient, en 1915, la protection du site et choisit d'imposer une couleur unique pour les boiseries des portes et des fenêtres. Ce bleu lumineux devenu aujourd'hui la couleur fétiche et le symbole de la Tunisie fut donc inventé par un aristocrate britannique. Depuis le XVIIIe siècle, les hauts fonctionnaires et les notables de Tunis s'étaient fait construire des résidences secondaires dans ce peitit village maraboutique dont l'origine 
remonte au XIIIe siècle. En effet, dès 1207, un ermite marocain, Abou Saïd Khalala ben Yahia el-Termini el-Beji (ouf !), vint se retirer ici pour vivre selon les principes du soufisme, entraînant dans sa retraite de nombreux disciples. Mort en 1236, il devint le patron du village (auquel il ne donna son nom qu'au XIXe siècle). Cependant, une autre version, à laquelle nous adhérons bien sûr, raconte qu'en fait Sidi-bou-Saïd serait Saint Louis ! Eh oui ! Lors de sa dernière croisade, fatigué, découragé, démoralisé par la peste décimant ses troupes, alors qu'il se promenait seul sur la plage, il tomba amoureux d'une belle Berbère. Pour pouvoir l'épouser, il fit croire à sa mort grâce à un sosie atteint de la peste. Il embrassa par la même occasion l'islam, et devint Sidi-bou-Saïd, ce saint vénéré aujourd'hui (bon! trop beau pour être vrai !).

Ce tombeau fut, pendant des siècles, le lieu de rencontre des membres des différentes confréries religieuses. Les corsaires devaient choisir Sidi-bou-Saïd comme patron de la piraterie contre les navires chrétiens. D'ailleurs, jusqu'en 1820, l'accès au village, haut lieu de spiritualité, fut interdit aux roumis. Ils prennent aujourd'hui leur revanche puisque l'on peut y compter en juillet et août jusqu'à 120000 visiteurs par jour.

[...]

\section{Où boire un verre?}

Le café des Nattes : le Sénéquier du coin (les Tropéziens comprendront...). Appelé Quahwa el-Alya (le " caféd'En-Haut ») par les habitants, c'est le troquet maure classique, dans la rue la plus touristique du village. Le thé à la menthe sur la terrasse ou à l'intérieur, allongé sur des nattes, est une étape quasi obligatoire. Ca fait quand même quelque chose de savoir qu'avant vous Gide, Montherlant, Bernanos, Paul Klee et Simone de Beauvoir, entre autres, se sont arrêtés ici pour y goûter un « instant de bonheur », et qu'André Gide venait y jouer aux dominos entre deux scènes d'Hamlet, drame shakespearien qu'il traduisit lors de son séjour en Tunisie. Bien entendu, la rue a changé depuis que Gustave Flaubert y rêvait de Salammbô.

Café Sidi-Chabaane : au bout de la rue principale et sur la droite ; impossible de vous tromper, suivez la foule qui s'y rend. En saison, à partir de 17 h, il devient parfois très difficile d'y trouver une place. Un endroit exceptionnel surplombant le golfe. Panorama magnifique dont on ne se lasse pas. Il faut observer le ballet des vendeurs de jasmin. On n'y sert que de la boisson et encore, le choix se limite à de la limonade, du café (excellent) et du thé à la menthe servi nature ou avec des pignons de pin (plus cher). Les Tunisiens viennent y fumer le chicha et restent des heures à observer les étoiles ou les lumières de la ville qui se reflètent sur la mer.

\section{NOTES}

1. Voir Annexe en fin d'article. Il comprend: deux "guides bleus », Hachette, Algérie, Tunisie (1955) GB1, Tunisie (1997) GB2, les guides «Visa », Hachette, En Tunisie (1996) GV, Berlitz Tunisie, guide de voyage (1998) B, Tunisie, Gallimard (1999) GG, "Neos", Michelin, Tunisie (2000) GN, Le grand guide de la Tunisie, Gallimard, (2000) GGT, « Guide du Routard », Hachette, (2000) GR.

2. GB1 est conforme à la tradition première des «Guides Bleus », caractérisés par le sérieux dû à la contribution de spécialistes des domaines traités, et il ne présente aucune autre icône que cartes et schémas architecturaux. Tous les autres guides, à l'exception de GR, présentent de nombreuses photos légendées; GG, GN et GGT se signalent par de longues parties informatives précédant les visites particulières ; GB2 est conforme aux «nouveaux » Guides bleus, allégés et illustrés. Le format de B et son nombre réduit de pages lui permettent de tenir facilement dans 
une poche. Quant à GR, il s'ouvre notamment sur «La Charte du routard». Chaque guide discrimine ainsi son lectorat, la même maison d'édition pouvant proposer plusieurs séries de guides.

3. Voir J.-J. Courtine (1981 : 53) : « La notion de mémoire discursive concerne l'existence historique de l'énoncé au sein de pratiques discursives [...] discours qui sont à l'origine d'actes nouveaux, de paroles qui les reprennent, les transforment ou parlent d'eux... ».

4. Voir Annexe : corpus.

5. Voir la troisième partie de l'article.

6. Pour J. Rey-Debove (1978), la connotation autonymique se caractérise par «le cumul de deux sémiotiques", le mot et la chose, et se glose en « comme on dit »; ici, la glose serait « comme on dit en arabe ».

7. La «modalisation autonymique » ou encore «modalisation opacifiante du dire » correspond aux mêmes phénomènes langagiers que ceux traités par J. Rey-Debove (1978) d'un point de vue sémiotique. L'approche retenue par J. Authier-Revuz (1995 et 2001) est énonciative. Voir aussi G. Petiot (2000).

8. C'est nous qui soulignons.

9. Larousse, 1994.

10. C'est nous qui soulignons. On peut constater que le classement opéré (le prototype) disparaît des reformulations (par d'autres guides), qui se contentent de caractériser le village comme «maraboutique ».

11. C'est nous qui soulignons.

12. Mais l'énoncé est complété par une suite qui invite à réviser la comparaison et donc à découvrir « le vrai visage, calme et paisible » du village, « le reste de l'année ». GV distingue ainsi deux villages, celui que les touristes voient «durant les mois d'été », à propos duquel le guide précise qu'il s'agit d'une « comparaison assez réaliste », et l'autre, à découvrir.

13. L'énoncé suivant semble corriger le bien fondé de la comparaison: «C'est un jugement un peu hâtif, autant dire faux. Si Sidi-Bou-Saïd est effectivement très touristique et hyper fréquenté en été, on se contente d'y passer quelques heures, mais qui valent le coup. L'infrastructure hôtelière, assez réduite, ne permet pas de séjour prolongé. » En fait, GR opposerait l'occupation permanente, l'été, des deux villages français, comparants, et celle de quelques heures par jour du village comparé tunisien.

14. C'est nous qui soulignons.

15. Modalité polysémique (« sans aucun doute », « peut-être » ?).

16. On peut relever l'absence de modalisation: la valeur aspectuelle /bornée/ du passé simple pose le fait comme indiscutable. Le soulignement est de notre fait.

17. Cet adjectif se trouve dans GB1, pour classifier l'art en général, l'architecture et la musique en particulier. On ne le retrouve guère que dans les descriptions du palais du baron d'Erlanger.

18. GGT titre ainsi les pages relatives à cette période : «La convoitise de l'Europe » (p.54) et inscrit dans « La Tunisie moderne » plusieurs pages (p.72-73) traitant de «La guerre du Golfe». L'histoire est convoquée de façon diversifiée, guide par guide, en fonction de la collection du guide.

19. Voir les travaux de J. Authier-Revuz. 


\section{AUTEUR}

\section{GENEVIÈVE PETIOT}

Paris 3 - Sorbonne Nouvelle, SYLED - CEDISCOR 